\title{
The Missing Link between Clinical States and Biomarkers in Mental Disorders
}

\author{
Giovanni A. Fava a b Jenny Guidi ${ }^{a}$ Silvana Grandi ${ }^{a}$ Gregor Hasler ${ }^{c}$ \\ a Laboratory of Psychosomatics and Clinimetrics, Department of Psychology, University of Bologna,

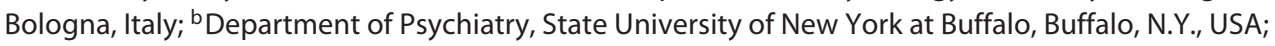 \\ 'Psychiatric University Hospital, University of Bern, Bern, Switzerland
}

\section{Key Words}

Biomarkers · Biological psychiatry · Clinical judgment .

Clinimetrics · Staging · Psychiatric diagnosis · Mental

disorders · Depressive disorder · Antidepressant agents .

Tolerance

\begin{abstract}
Current diagnostic definitions of psychiatric disorders based on collections of symptoms encompass very heterogeneous populations and are thus likely to yield spurious results when exploring biological correlates of mental disturbances. It has been suggested that large studies of biomarkers across diagnostic entities may yield improved clinical information. Such a view is based on the concept of assessment as a collection of symptoms devoid of any clinical judgment and interpretation. Yet, important advances have been made in recent years in clinimetrics, the science of clinical judgment. The current clinical taxonomy in psychiatry, which emphasizes reliability at the cost of clinical validity, does not include effects of comorbid conditions, timing of phenomena, rate of progression of an illness, responses to previous treatments, and other clinical distinctions that demarcate major prognostic and therapeutic differences among patients who otherwise seem to be deceptively sim-
\end{abstract}

\section{KARGER}

E-Mail karger@karger.com

www.karger.com/pps ilar since they share the same psychiatric diagnosis. Clinimetrics may provide the missing link between clinical states and biomarkers in psychiatry, building pathophysiological bridges from clinical manifestations to their neurobiological counterparts.

(c) 2014 S. Karger AG, Base

Kapur et al. [1] deserve credit for acknowledging the failure of biological psychiatry to produce clinical tests that could be routinely used in diagnosis and treatment of mental disorders. The impressive body of papers and books that have been produced in the past decades does not seem to have yielded clinically relevant findings. Kapur et al. [1] suggest that, rather than seeking medical tests that can diagnose DSM-defined disorders, the field should focus on identifying biologically homogeneous subtypes that cut across phenotypic diagnoses in what is termed 'stratified psychiatry'. The Research Domain Criteria launched by the National Institute of Mental Health [2] attempt to unravel neurobiological systems and genetic predisposition for subtyping current disorders and ultimately for overcoming current diagnostic systems. However, while the National Institute of Mental Health policy rightfully emphasizes the need for pilot investiga-
C 2014 S. Karger AG, Basel

0033-3190/14/0833-0136\$39.50/0
G.A. Fava, MD

Department of Psychology

Viale Berti Pichat 5

IT-40127 Bologna (Italy)

E-Mail giovanniandrea.fava@ unibo.it 
tions for supporting large trials, there is no evidence to support this new lead. Indeed, Kapur et al. [1] were unable to provide exemplifications suggesting that this approach was likely to yield meaningful clinical results in psychiatry. The evidence may come from other fields of medicine, with the underlying assumption that the same phenomena are operational. Their illustration of this approach is rich in intellectual fascination [1, p. 1174]:

Thus, patients who in early classifications would be noted to have 'dropsy and dyspnea' were successfully subjected to listening of their murmurs with a stethoscope, to picturing of their enlarged heart in a chest X-ray, to recording of their arrhythmias with an electrocardiogram, to calculation of their ejection fractions with a 2-D Echo and increasingly to a series of new biomarkers (for example atrial natriuretic factor) that lead to a more refined diagnosis and targeted treatment.

Actually, things are not that simple also in cardiology [3]. Most meta-analyses of biomarkers commonly used in cardiovascular medicine show evidence of publication bias and selective reporting [4]. Biomarkers may be the result of different mechanisms, not necessarily reflecting a specific disease process [5]. The complexity of the brain and the spurious nature of measurements that can be recorded constitute a major difficulty for psychiatry. Specifically, the neuroplastic properties make the brain a unique organ that essentially has to be studied and understood in a longitudinal, lifetime and transgenerational perspective [6]. In contrast, Kapur et al. [1] view assessment essentially as a cross-sectional collection of reported symptoms and do not pay attention to the use of a stethoscope in psychiatry.

\section{Is There a Stethoscope in the House?}

This was the title of a classic paper by McIntyre and Romano [7]. It reported on the attitudes of psychiatrists concerning the physical examination of psychiatric patients. The majority of psychiatrists did not perform and/ or did not feel competent to perform physical examinations. A renewed interest in the medical aspects of psychiatry sparked a search for undetected medical illness in psychiatric patients that might also be contributing to psychiatric symptomatology [8]. The importance of detecting medical disorders in psychiatric patients was a major drive in the introduction of the category of organic affective syndrome in DSM-III led by Lipowski [9]. With this diagnostic definition one could postulate that a depressive or manic syndrome could be induced by direct interference with the neurophysiological and biochemi- cal processes subserving normal mood $[9,10]$. The hypothesis was compatible with the view that causes of depressive disorders may be heterogeneous [11] and was rich in research implications. For instance, the clinical observation of resistance to antidepressant drugs in depression occurring in Cushing's syndrome and its responsiveness to steroid production inhibitors [12] paved the way for the use of these latter drugs in psychiatry [13]. Similarly, some characteristics of depression in Cushing's syndrome may have pathophysiological implications for the melancholic and atypical subtypes of depression in psychiatry in relation to characteristics of the hypothalamic-pituitary-adrenal axis [14].

The classic stethoscope a cardiologist uses, however, is not the only one that psychiatrists may employ.

\section{The Role of Clinical Judgment}

In their everyday practice, psychiatrists use observation, description and classification, test explanatory hypotheses, and formulate clinical decisions [15]. In evaluating whether a patient needs admission to the hospital (or can be discharged from it), in deciding whether a patient needs treatment (and in case what type) and in planning the schedule of follow-up visits or interventions, the psychiatrist uses nothing more than the science of psychopathology [16] and clinical judgment [15].

In 1982, Feinstein introduced the term 'clinimetrics' [17] to indicate a domain concerned with the measurement of clinical issues that do not find room in customary clinical taxonomy. Such issues include the types, severity and sequence of symptoms; rate of progression in illness (staging); severity of comorbidity; problems of functional capacity; reasons for medical decisions (e.g. treatment choices), and many other aspects of daily life, such as well-being and distress $[18,19]$. The customary clinical taxonomy in psychiatry does not include patterns of symptoms, severity of illness, effects of comorbid conditions, timing of phenomena, rate of progression of illness, responses to previous treatments, and other clinical distinctions that demarcate major prognostic and therapeutic differences among patients who otherwise seem to be deceptively similar since they share the same psychiatric diagnosis [20]. For instance, the main difference between schizophrenia and bipolar disorder, if such a difference exists at all, may not be their symptom presentations and abnormally functioning neurocircuitry but their distinguishable patterns of illness progression. The risk factor profile for major depressive disorder changes consider- 
ably in the course of the illness [21]. As a result, clinimetric research in psychiatry will yield important insights into neurobiologically and genetically relevant clinical judgments [20, 22].

A first strategy is concerned with staging. It differs from the conventional diagnostic practice in that it does not only define the extent of progression of a disorder at a particular point in time, but also where a person is currently along the continuum of the course of illness (longitudinal development), and his/her treatment history [23]. Neurobiological markers may be different according to the stages of development of disorders. Staging methods for unipolar depression, bipolar disorder, panic disorder and schizophrenia and other disturbances have been developed [23, 24] after their introduction in 1993 [25]. In two randomized controlled trials [26, 27], psychotherapeutic intervention was applied according to a staging method and was found to yield long-term benefits $[28,29]$. Such studies underwent independent replication [30].

A second approach involves building unitary concepts from apparently scattered phenomena. Tyrer et al. [31] remarked that what is shared by syndromes such as anxiety, panic, phobic disturbances and irritability may be as important as the differences between them and conditions that are apparently comorbid could be part of the same clinical syndrome. They argued that the combination of mixed anxiety and depressive disorders together with a certain type of abnormal personality, constitute a single syndrome, the general neurotic syndrome [31]. The syndrome was shown to be associated with a poor response to treatment, frequent symptoms throughout the neurotic diagnostic spectrum and tendency to relapse [31]. A related strategy deals with the concept of allostatic load, the cumulative effects of stressful experiences in daily life [32]. There have been several attempts to identify allostatic load by the use of laboratory biological markers, such as 12-hour urinary cortisol, blood dehydroepiandrosterone and its sulfated form, high-density lipoprotein and total cholesterol, glycosylated hemoglobin, and proinflammatory cytokines, as well as clinical parameters, such as systolic and diastolic blood pressure, and waist-hip ratio [33]. The results, however, have been rather disappointing, not only because these markers do not come close to a comprehensive measure of dysregulation in the whole organism [34], but also because they are nonspecific mediators and may be affected by other variables that change from one individual to another. Using clinimetric principles, Fava et al. [32] have introduced specific clinical criteria for the assessment of allostatic overload, a state due to the cumulative interactions between life events and chronic life stressors that, by exceeding the individual resources, may constitute a danger to health. The application of the clinimetric criteria for allostatic overload [32] has yielded promising results in recent studies conducted both in the general population $[35,36]$ and in the setting of cardiovascular diseases [37, 38 ], with particular reference to their capacity to discriminate immune biomarkers, health variables and psychopathological measures.

A complementary strategy has to do with subtyping and differentiating within a diagnostic entity. The need for subtyping major depressive disorder, since this category is too broad to yield meaningful treatment implications, has recently been underscored [39-43]. The basic assumption is that clinical manifestations that share the diagnosis of major depressive disorder may display substantial differences in prognostic and therapeutic terms [39-41]. Careful symptom discrimination by interviewing may allow to attribute differential emphasis on specific symptoms. In clinimetrics, major and minor symptoms may be discriminated, unlike in psychometrics where all items have equal weighting [19].

A fourth approach entails the consideration of treatment history. The standard randomized controlled trial design is still based on the acute disease model and ideally evaluates therapeutic effects in untreated patients who have a recent acute onset of their disturbances [44]. This is in sharp contrast to the fact that, particularly in psychopharmacology, the patient is likely to have experienced other treatments before and these treatments may actually modify the course and responsiveness of the individual patient [45]. Under normal circumstances, patients are included in a trial regardless of their treatment history. The heterogeneous features of these patients would then affect the outcome of the trial. An example may be provided by the problems related to the loss of clinical effect during long-term antidepressant treatment [45]. The recurrence of depressive symptoms during maintenance antidepressant treatment was found to occur in $9-57 \%$ of patients in published trials [46]. Pharmacological tolerance, loss of placebo effect, increase in disease severity, change in disease pathogenesis, accumulation of a detrimental metabolite, unrecognized rapid cycling, and prophylactic inefficacy have been suggested as possible explanations [46]. There is virtually no exploration of the neurobiological correlates of the loss of clinical effect, despite its clinical importance and the practical implications that research in this area would entail [45]. Emphasis is placed on constructs such as treatment-resis- 
tant depression that do not discriminate clinical phenomena such as loss of clinical effect. The conceptual assumption is that after testing a standard treatment in a group of patients we are left with a fairly homogeneous group characterized by resistance. Actually, nonresponse includes a very wide range of explanations (inadequate treatment in terms of duration, doses or indications; the occurrence of side effects prevailing over benefits; partial compliance; previous exposure to that specific treatment; psychosocial events intervening during the trial; problems in the patient-doctor relationship; modifications in patient's lifestyle and illness behavior). As a result, designs that use highly heterogeneous populations at the start and are based on identification of nonresponse follow an oversimplified conceptual model that clashes with the clinical reality they would attempt to mimic [47]. In schizophrenia research, the interpretation of gray matter volumes in terms of 'biotypes of psychosis' may lead to questionable conclusions, given that long-term treatments with antipsychotics lead to important progressive brain changes [48]. Likewise, the simplistic assumption that neurocognitive impairment is the core of the disorder may prove wrong in high-functioning schizophrenic patients with predominant affective dysregulation. In fact, the clinical relevance of one-time 'objective' neurocognitive tests (and their neurobiological and genetic underpinnings) may be quite limited in many nonneurodegenerative psychiatric conditions, since it is the intraindividual change in cognitive performance rather than its position in the distribution in the general population that is associated with personal suffering. In depression, the amount of research attempting a neurobiological characterization of cross-sectionally assessed depressive symptoms is immense. But what is its translational value?

\section{The Intellectual Crisis of Psychiatric Research}

Engel [49] differentiated between 'scientific physicians' (clinicians who fully apply the scientific method in their care of patients and in their understanding of the disease) and 'physician-scientists' (physicians whose primary commitment is to scientific research pertaining to medicine and who have little or no familiarity with the clinical process). Clinical practice is the source of fundamental scientific challenges for scientific physicians, whereas the application of basic (including pharmaceutical) research is the preferred focus of physician-scientists. Part of the challenge and, at the same time, fascination of being a clinician lies in applying scientific methods in the care of patients and in understanding disease [50]. Greater knowledge should result in significant benefits for the patients, and in a sense of continued development on the part of the physician. We are witnessing, however, a progressive detachment of clinicians from research [49-51].

Feinstein [51] attributed the main root of this development to the destruction of the pathophysiological bridges from bench to bedside and the fact that clinicians were thus urged to apply models derived from basic domains such as molecular biology or economics. Clinical observation is no longer the fundamental source of scientific challenges. 'All the fundamental scholarly ideas come from elsewhere, and clinicians apparently have nothing important to contribute beyond their work in applying the basic ideas' [51, p. 217]. Neurosciences have exported their conceptual framework into psychiatry much more than serving as an investigative tool for addressing the questions entailed by clinical practice. Not surprisingly, there is a very limited amount of clinically relevant findings [52-54].

Yet, by ignoring the pathophysiology of clinical manifestations that can often be captured only by clinical judgment, neurobiological research is missing important opportunities for practical advances in the application of biomarkers. Use of clinical history and outcome data may enhance the utility of biomarkers [5] and may provide iteration in the validation process [55].

In the same vein, the effects of comorbidity on the clinical course of an index disease (a major challenge in biological psychiatry research) cannot be determined on the basis of a simple data collection and statistical analysis of a large number of patients. It requires acts of clinical reasoning and incorporation of treatment history. Feinstein's [56, pp. 456-457] classic definition of comorbidity as 'any distinct additional clinical entity that has existed or that may occur during the clinical course of a patient who has the index disease under study' referred also to antecedent pathological events that were judged to affect current disease. The cross-sectional nature of the classification systems in psychiatry has limited the use of the term 'comorbidity' to what a patient may be currently experiencing. Likewise, emphasis on short-term evaluations of the efficacy of psychotropic drugs required by regulators such as the Food and Drug Administration does not sufficiently consider the long-term course and importance of relapse prevention in mental illness. Such mainstream views are not taking into account the fundamentally neuroplastic nature of the brain, the significance of psychological and somatic pathogenetic events in a longitudinal perspective, the sequences of gene-environ- 
ment interactions paving the way from health to disease, the pathogenetic role of epigenetic and transgenerational effects, and the long-term effects of pharmacotherapy and psychosocial interventions.

\section{Conclusions}

An increasing number of psychiatrists are wondering why the cures and clinical insights that neurosciences have promised have not become available. Biological reductionism [50] has resulted in an idealistic approach, which is quite far from the explanatory pluralism required by clinical practice. Kendler [57], Van Praag [58] and Belmaker [59] have been outspoken critics of this reductionism.

Engel [60] identified the key characteristic of clinical science in its explicit attention to humanness, where observation (outer viewing), introspection (inner viewing) and dialogue (inter-viewing) are the basic methodological triad for clinical assessment and for making patient data scientific. The exclusion of this interaction by medical science's continuing allegiance to a 17 th century scientific world view makes this approach unscientific. Unlike 20th century physics, 'the human realm either has been excluded from accessibility to scientific inquiry or the scientific approach to human phenomena has been required to conform to the reductionistic, mechanistic, dualistic predicates of the biomedical paradigm' [60]. This restrictive ideology [61] characterizes the Research Domain Criteria [2]. It is time to enrich such criteria with clinically relevant dimensions and add clinical validity to the reliability and reductionism-focused mainstream of psychiatry research.

In 1967, Feinstein [62] urged clinicians to develop a 'basic science' on their own to study the clinical phenomena directly, to specify the importance of different types of clinical data, to create appropriate systems of taxonomy for classifying the information, and to develop intellectual models and pragmatic methods that would articulate the clinical process and use the results for quantified analyses. Such a line of research, that affects clinical decision making, has produced clinically incisive developments in psychiatry [20]. It is time to substitute the fashionable popularity of strategies developed outside of psychiatry with creative neurobiological research based on the insights of clinical judgment.

\section{Disclosure Statement}

The authors have no conflict of interest to declare.

\section{References}

1 Kapur S, Phillips AG, Insel TR: Why has it taken so long for biological psychiatry to develop clinical tests and what to do about it? Mol Psychiatry 2012;17:1174-1179.

-2 Insel T, Cuthbert B, Gavery M, Heinssen R, Pine DS, Quinn K, Sanislow C, Wang P: Research Domain Criteria (RDoC): toward a new classification framework for research on mental disorders. Am J Psychiatry 2010;167: 748-751.

-3 Nissen SE: Biomarkers in cardiovascular medicine: the shame of publication bias. JAMA Intern Med 2013;173:671-672.

-4 Tzoulaki I, Siontis KC, Evangelou E, Ioannidis JPA: Bias in associations of emerging biomarkers with cardiovascular disease. JAMA Intern Med 2013;173:664-671

5 Woodcock J: Assessing the clinical utility of diagnostics used in drug therapy. Clin Pharmacol Ther 2010;88:765-773.

6 Kellermann NP: Epigenetic transmission of holocaust trauma: can nightmares be inherited? Isr J Psychiatry Relat Sci 2013;50:33-37.
7 McIntyre JS, Romano J: Is there a stethoscope in the house (and is it used)? Arch Gen Psychiatry 1977;34:1147-1151.

8 Koranyi EK, Potoczny WM: Physical illnesses underlying psychiatric symptoms. Psychother Psychosom 1992;58:155-160.

9 Lipowski ZJ: Organic mental disorders: introduction and review of syndromes; in Kaplan HI, Freedman AM, Sadock BJ (eds): Comprehensive Textbook of Psychiatry/III. Baltimore, Williams \& Wilkins, 1980, pp 13591392.

10 Fava GA, Sonino N: Depression associated with medical illness: treatment implications. CNS Drugs 1996;5:175-189.

11 Winokur G: All roads lead to depression: clinically homogeneous, etiologicall heterogeneous. J Affect Disord 1997;45:97-108.

12 Sonino N, Fallo F, Fava GA: Psychosomatic aspects of Cushing's syndrome. Rev Endocr Metab Disord 2010;11:95-104.

13 Reus VI, Wolkowitz OM: Antiglucocorticoid drugs in the treatment of depression. Expert Opin Investig Drugs 2001;10:17891796.
14 Gold PW, Chrousos P: Melancholic and atypical subtypes of depression represent distinct pathophysiological entities. Mol Psychiatry 2013;18:632-634.

15 Fava GA: Clinical judgment: requiem or reveille? Nord J Psychiatry 2013;67:1-10.

16 Lipowski ZJ: Psychopathology as a science: its scope and tasks. Comp Psychiatry 1966;7: 175-182.

17 Feinstein AR: The Jones criteria and the challenges of clinimetrics. Circulation 1982;66: $1-5$.

18 Feinstein AR: Clinimetrics. New Haven, Yale University Press, 1987.

19 Fava GA, Tomba E, Sonino N: Clinimetrics: the science of clinical measurements. Int J Clin Practice 2012;66:11-15.

20 Fava GA, Rafanelli C, Tomba E: The clinical process in psychiatry: a clinimetric approach. J Clin Psychiatry 2012;73:177-184.

21 Kendler KS, Thornton LM, Gardner CO: Stressful life events and previous episodes in the etiology of major depression in women: an evaluation of the 'kindling' hypothesis. Am J Psychiatry 2000;157:1243-1251. 
-22 Tomba E, Bech P: Clinimetrics and clinical psychometrics. Psychother Psychosom 2012; 81:333-343.

23 Cosci F, Fava GA: Staging of mental disorders: systematic review. Psychother Psychosom 2013;82:20-34.

24 McGorry P, Hickie IB, Yung AR, Pantelis C, Jackson $\mathrm{HJ}$ : Clinical staging of psychiatric disorders. Aust N Z J Psychiatry 2006;40:612622.

25 Fava GA, Kellner R: Staging: a neglected dimension in psychiatric classification. Acta Psychiatr Scand 1993;87:225-230.

-26 Fava GA, Grandi S, Zielezny M, Canestrari R, Morphy MA: Cognitive behavioral treatment of residual symptoms in primary major depressive disorder. Am J Psychiatry 1994;151: 1295-1299.

27 Fava GA, Rafanelli C, Grandi S, Conti S, Belluardo P: Prevention of recurrent depression with cognitive behavioral therapy. Arch Gen Psychiatry 1998;55:816-820.

28 Fava GA, Grandi S, Zielezny M, Rafanelli C, Canestrari R: Four year outcome for cognitive behavioral treatment of residual symptoms in major depression. Am J Psychiatry 1996;153: 945-947.

-29 Fava GA, Ruini C, Rafanelli C, Finos L, Conti S, Grandi S: Six-year outcome of cognitive behavior therapy for prevention of recurrent depression. Am J Psychiatry 2004;161:18721876.

-30 Guidi J, Fava GA, Fava M, Papakostas GI: Efficacy of the sequential integration of psychotherapy and pharmacotherapy in major depressive disorder: a preliminary meta-analysis. Psychol Med 2011;41:321-331.

- 31 Tyrer P, Seivewright N, Ferguson B, Tyrer J: The general neurotic syndrome. Acta Psychiatr Scand 1992;85:201-206.

-32 Fava GA, Guidi J, Semprini F, Tomba E, Sonino $\mathrm{N}$ : Clinical assessment of allostatic load and clinimetric criteria. Psychother Psychosom 2010;79:280-283.

33 McEwen BS: Central effects of stress hormones in health and disease: understanding the protective and damaging effects of stress and stress mediators. Eur J Pharmacol 2008; 583:174-185.
34 McEwen BS: Physiology and neurobiology of stress and adaptation: central role of the brain. Physiol Rev 2007;87:873-904.

35 Offidani E, Ruini C: Psychobiological correlates of allostatic overload in a healthy population. Brain Behav Immun 2012;26:284-291.

36 Tomba E, Offidani E: A clinimetric evaluation of the allostatic overload in the general population. Psychother Psychosom 2012;81:378379.

37 Offidani E, Rafanelli C, Gostoli S, Marchetti G, Roncuzzi R: Allostatic overload in patients with atrial fibrillation. Int J Cardiol 2012;165: 375-376.

38 Porcelli P, Laera D, Mastrangelo D, Di Masi A: Prevalence of allostatic overload syndrome in patients with chronic cardiovascular disease. Psychother Psychosom 2012;81:375377.

39 Lichtenberg P, Belmarker RH: Subtyping major depressive disorder. Psychother Psychosom 2010;79:131-135.

40 Bech P: The struggle for subtypes in primary and secondary depression and their modespecific treatment or healing. Psychother Psychosom 2010;79:331-338.

41 Guidi J, Fava GA, Picardi A, Porcelli P, Bellomo, Grandi S, Grassi L, Pasquini P, Quartesan R, Rafanelli C, Rigatelli M, Sonino N: Subtyping depression in the medically ill by cluster analysis. J Affect Disord 2011;132. 383-388.

42 Hasler G, Drevets WC, Manji H, Charney DS: Discovering endophenotypes for major depression. Neuropsychopharmacology 2004; 29:1765-1782.

43 Hasler G, Northoff G: Discovering imaging endophenotypes for major depression. Mol Psychiatry 2011;16:604-619.

44 Tomba E: Nowhere patients. Psychother Psychosom 2012;81:69-72.

45 Fava GA, Offidani E: The mechanisms of tolerance in antidepressant action. Prog Neuropsychopharmacol Biol Psychiatry 2011;35: 1593-1602.

46 Byrne SE, Rotschild AJ: Loss of antidepressant efficacy during maintenance therapy. J Clin Psychiatry 1998;59:279-288.
47 Fava GA, Tomba E, Tossani E: Innovative trends in the design of therapeutic trials in psychopharmacology and psychotherapy. Prog Neuropsychopharmacol Biol Psychiatry 2013;40:306-311

48 Fusar-Poli P, Radua J, Frascarelli M, Mechelli A, Borgwardt S, Di Fabio F, Biondi M, Ioannidis JP, David SP: Evidence of reporting biases in voxel-based morphometry (VBM) studies of psychiatric and neurological disorders. Hum Brain Mapp 2013, DOI: 10.1002/ hbm.22384.

49 Engel GL: Physician scientists and scientific physicians. Am J Med 1987;82:107-111.

50 Fava GA: The intellectual crisis of psychiatric research. Psychother Psychosom 2006;75: 202-208.

51 Feinstein AR: The intellectual crisis in clinical science. Perspect Biol Med 1987;30:215-230.

52 Hasler G: Pathophysiology of depression: do we have any solid evidence of interest to clinicians? World Psychiatry 2010;9:155-161.

53 Balon R: Clinical factor 2011. Psychother Psychosom 2012;81:199-205.

54 Balon R: Clinical factor 2012. Psychother Psychosom 2013;82:204-212.

55 Carroll BJ: Biomarkers in DSM-5:lost in translation. Aust N Z J Psychiatry 2013;47: 676-681.

56 Feinstein AR: The pre-therapeutic classification of comorbidity in chronic disease. J Chron Dis 1970;23:455-468.

57 Kendler KS: Toward a philosophical structure for psychiatry. Am J Psychiatry 2005;162: 433-440.

58 Van Praag HM: Over the mainstream: diagnostic requirements for biological psychiatry research. Psychiatry Res 1997;72:201-212.

59 Belmaker RH: The future of depression psychopharmacology. CNS Spectr 2008;13:682687.

60 Engel GL: How much longer should medicine's science be bound by a seventeenth century world view? Psychother Psychosom 1992;57:3-16.

-61 Fava GA, Ruini C, Tomba E, Wise TN: The biopsychosocial factor. Psychother Psychosom 2012;81:1-4.

62 Feinstein AR: Clinical Judgment. Baltimore, Williams \& Wilkins, 1967. 